\title{
Osteoclastogenesis accompanying early osteoblastic differentiation of BMSCs promoted by mechanical stretch
}

\author{
YUQIONG WU, PENG ZHANG, QINGGANG DAI, RUNQING FU, \\ XIAO YANG, BING FANG and LINGYONG JIANG
}

\begin{abstract}
Center of Craniofacial Orthodontics, Department of Oral and Cranio-Maxillofacial Science, Ninth People's Hospital, Shanghai Jiao Tong University School of Medicine, Shanghai Key Laboratory of Stomatology, Shanghai 200011, P.R. China
\end{abstract}

Received January 21, 2013; Accepted March 11, 2013

DOI: $10.3892 /$ br.2013.84

\begin{abstract}
Mechanical stress plays a crucial role in bone formation and absorption. In previous studies, we verified the osteoblastogenesis of bone mesenchymal stem cells (BMSCs) affected by intermittent traction stretch. However, little is known about the osteoclastogenesis process under mechanical stimulation and its underlying association with osteoblastogenesis. In the present study, we investigated the osteoclastogenesis of BMSCs under this special mechanical stress. BMSCs were subjected to $10 \%$ elongation for 1-7 days using a Flexcell Strain Unit and then the mRNA levels of osteoclastic genes were examined. The results indicated time-dependent varying of mRNA levels of the receptor activator of nuclear factor- $\kappa \mathrm{B}$ ligand (RANKL) and osteoprotegerin (OPG) in BMSCs at different stretching time points. The ratio of RANKL/OPG increased at the early stage of mechanical stimulation (5 days) and decreased to a low level at a later stage (7 days). Findings of this study may help to understand the correlations between osteoblastogenesis and osteoclasteogenesis when mechanical stretch induces the osteoblastic differentiation of BMSCs.
\end{abstract}

\section{Introduction}

Skeleton is a highly organized tissue that provides a structural framework to facilitate the locomotion and activity of daily living. As such, it is metabolically active and is able to sense and adapt to mechanical stimuli in order to maintain a balance between bone formation and absorption (1). During orthodontic

Correspondence to: Dr Lingyong Jiang or Dr Bing Fang, Center of Craniofacial Orthodontics, Department of Oral and Cranio-Maxillofacial Science, Ninth People's Hospital, Shanghai Jiao Tong University School of Medicine, Shanghai Key Laboratory of Stomatology, Room 405, Building 1, 639 Zhi Zao Ju Road, Shanghai 200011, P.R. China

E-mail: jly117@sina.com

E-mail: fangbing@sjtu.edu.cn

Key words: mechanical stretch, bone mesenchymal stem cells, receptor activator of nuclear factor- $\kappa \mathrm{B}$ ligand, osteoprotegerin, osteoclastogenesis tooth movement, mechanical stimuli also play a crucial role in bone remodeling as a result of osteoblast and osteoclast.

Physiologically, tooth movement is a slow process that occurs mainly in the buccal direction into cancellous bone. By contrast, orthodontic tooth movement can occur rapidly or slowly, depending on the physical characteristics of the applied force and the size and biological response of the periodontal ligament (PDL). This tooth movement has been defined as the result of a biologic response to interference in the physiologic equilibrium of the dentofacial complex by an externally applied force (2), which generates two different strains in the PDLs, compression and tension. At the compression site, the force that is generated by the root against the alveolar bone induces bone resorption. At the tension site, PDL fibers are stretched and bone tissue is formed $(3,4)$. Alveolar bone formation results from complex events that involve the differentiation of osteoblast precursor cells from primitive bone mesenchymal stem cells (BMSCs), maturation of osteoblasts, and matrix formation, followed by its mineralization (4-6). Nevertheless, several lines of evidence $(7,8)$ suggest that cells of the osteoblastic lineage are involved in osteoclast formation. However, the role played by orthodontic stretch regulating osteoclastogenesis induced by BMSCs during initial osteoblastic differentiation in alveolar bone remains to be determined.

Research in osteoclast differentiation has been greatly advanced since the identification of the receptor activator of nuclear factor- $\kappa \mathrm{B}$ ligand (RANKL) as an osteoclast differentiation factor. RANKL was identified as a key cytokine that binds to its receptor on osteoclast progenitors, and then stimulates the activity and recruitment $(7,8)$. Osteoprotegerin (OPG) has been identified as a competitive receptor of RANKL that may prevent the activation of RANK resulting in decreased osteoclast activity (9). This association between osteoclasts and osteoblasts in bone metabolism is greatly affected by the balance between RANKL and OPG, which modulates the level of bone resorption on bone surfaces.

In a previous study, we found that exposure to intermittent traction stretch induced osteoblastic differentiation of BMSCs $(10,11)$. However, whether stretch-promoted osteoblastic differentiation of BMSCs also affected osteoclastogenesis remains to be elucidated. The present study was designed to investigate the association of osteoclastogenesis with osteoblastic differentiation of BMSCs. By evaluating 
the effects of mechanical stretch on BMSCs insight may be gained into the mechanisms of both bone regeneration and orthodontic movement in vivo.

\section{Materials and methods}

Isolation and identification of rBMSCs. The tibia and femur were isolated from $160 \pm 10 \mathrm{~g}$, male Sprague-Dawley rats (Shanghai SLAC Experimental Animal Center, Shanghai, China). The bone marrow was flushed out with $\alpha$-minimal essential medium ( $\alpha$-MEM; Hyclone, Thermo, USA) supplemented with $100 \mathrm{U} / \mathrm{ml}$ penicillin, and $100 \mu \mathrm{g} / \mathrm{ml}$ streptomycin (Hyclone). To remove the blood cells, the whole washouts were collected and centrifuged at 1,800 x $\mathrm{g}$ for $10 \mathrm{~min}$. The precipitate was mixed with complete $\alpha$-MEM supplemented with $10 \%$ fetal bovine serum (FBS, Hyclone), $100 \mathrm{U} / \mathrm{ml}$ penicillin and $100 \mu \mathrm{g} / \mathrm{ml}$ streptomycin and then plated into a culture flask maintained at $37^{\circ} \mathrm{C}$ in $5 \% \mathrm{CO}_{2}$. Non-adherent cells were removed by changing the medium every 3 days for $\sim 10$ days. When large colonies formed and became confluent, the primary rat BMSCs were trypsinized with $10 \%$ trypsin-EDTA (Hyclone) and passaged. Analysis of cell surface molecules was performed on passages 2 using flow cytometry for CD31, CD44 and CD90. BMSCs from passages 2-5 were used for subsequent experiments.

Alkaline phosphatase (ALP) quantification assay. To observe the osteoblastic differentiation characteristic of BMSCs, cells were cultured either in the growth or osteogenic medium, comprising Dulbecco's modified Eagle's medium (DMEM, Hyclone) supplemented with $10 \%$ FBS, $10 \mathrm{mM} \beta$-glycerophosphate, $100 \mathrm{nM}$ dexamethasone, and $50 \mathrm{M}$ ascorbic acid (all from Sigma-Aldrich, St. Louis, MO, USA). BMSCs were rinsed twice with ice-cold PBS, scraped from the dishes and suspended in $\mathrm{ddH}_{2} \mathrm{O}$. This was followed by three cycles of freezing and thawing. ALP activity was determined at $405 \mathrm{~nm}$ using p-nitrophenyl phosphate (pNPP) (Sigma-Aldrich) as the substrate. A $50 \mu \mathrm{l}$ of sample was mixed with $50 \mu$ of pNPP $(1 \mathrm{mg} / \mathrm{ml})$ in $1 \mathrm{M}$ diethanolamine buffer containing $0.5 \mathrm{mM} \mathrm{MgCl} 2(\mathrm{pH} 9.8)$ and incubated at $37^{\circ} \mathrm{C}$ for $15 \mathrm{~min}$ on a bench shaker. The reaction was stopped by the addition of $200 \mu \mathrm{l}$ of $2 \mathrm{M} \mathrm{NaOH}$ per $200 \mu \mathrm{l}$ of reaction mixture. Total protein content was determined by the BCA method using a protein assay kit (Pierce, Rockford, IL, USA). ALP activity was calculated as nmol p-nitrophenol per minute per mg protein, and presented as fold changes over the un-induced group at the respective time points. The experiments were conducted in triplicate.

Mechanical tension load application. BMSCs were plated at a density of $1 \times 10^{5}$ cells $/ \mathrm{ml}$ (unless stated otherwise) in $2 \mathrm{ml}$ of medium on six-well flexible silicone rubber BioFlex ${ }^{\mathrm{TM}}$ plates coated with collagen type I (Flexcell International Corp., Hillsborough, NC, USA). The cells were cultured for $48 \mathrm{~h}$ to allow them to attach and reach $80-90 \%$ confluency, at which time the growth medium was replaced, and mechanical strain was applied. A cyclic mechanical strain with a $0.5 \mathrm{~Hz}$ sinusoidal curve set at $10 \%$ elongation was applied twice every day, for $4 \mathrm{~h}$ for each treatment, using a FX-4000T ${ }^{\text {тм }}$ Flexcell Tension Plus $^{\mathrm{TM}}$ unit (Flexcell International Corp.). The cultures
Table I. List of primers used and their respective forward and reverse sequences.

\begin{tabular}{ll}
\hline Gene & \multicolumn{1}{c}{ Sequence } \\
\hline$\beta$-actin & F: 5'-GTAAAGACCTCTATGCCAACA-3' \\
& R: 5'-GGACTCATCGTACTCCTGCT-3' \\
OPG & F: 5'-TTGAAATGGCAGTTGATTCCTTT-3' \\
& R:5'-TATCCTCTTTCTCAGGGTGCTTG-3' \\
RANK ligand & F: 5'-CATCGGGTTCCCATAAAG-3' \\
& R: 5'-GAAGCAAATGTTGGCGTA-3' \\
\hline
\end{tabular}

OPG, osteoprotegerin; RANK, receptor activator of nuclear factor- $\kappa \mathrm{B}$.

were incubated in a humidified atmosphere at $37^{\circ} \mathrm{C}$ and $5 \%$ $\mathrm{CO}_{2}$ while stretching. The BMSCs were collected after 1, 3, 5 and 7 days of stretch stimulation.

ALP staining. The presence of ALP in the cell layers was assessed according to the manufacturer's instructions (Beyotime, Suzhou, China). The BMSCs from the control and stretch treatment groups were rinsed with PBS three times and fixed with $4 \%$ paraformaldehyde for $10 \mathrm{~min}$. The fixed cells were soaked in $0.1 \%$ naphthol AS-MX phosphate and $0.1 \%$ fast red violet LB salt in $56 \mathrm{mM}$ 2-amino-2-methyl-1, 3-propanediol for $45 \mathrm{~min}$ at $37^{\circ} \mathrm{C}$, washed with $\mathrm{ddH}_{2} \mathrm{O}$, and then visualized with a digital camera.

Real-time PCR. The total RNA of the cells was isolated using the TRIzol reagent (Invitrogen, Carlsbad, CA, USA), according to the manufacturer's instructions. The RNA concentrations were determined using a NanoDrop spectrophotometer (Thermo Scientific, Wilmington, DE). Complimentary DNA (cDNA) was synthesized by means of a cDNA Synthesis Reverse Transcription kit (Fermentas, Thermo Scientific, Wilmington, DE, USA). Real-time PCR was performed using an ABI 7900HT system with SYBR Premix Ex Taq ${ }^{\mathrm{TM}}$ (Takara, Dalian, China), according to the manufacturer's instructions. The conditions of the real-time PCR were as follows: denaturation at $95^{\circ} \mathrm{C}$ for $10 \mathrm{sec} ; 50$ cycles at $95^{\circ} \mathrm{C}$ for $10 \mathrm{sec}$ and $60^{\circ} \mathrm{C}$ for $30 \mathrm{sec}$; and a final dissociation stage $\left(95^{\circ} \mathrm{C}\right.$ for $\left.5 \mathrm{~min}\right)$ was added at the end of the amplification procedure. $\beta$-actin was used as an internal control. The data were analyzed using the comparative $\mathrm{Ct}\left(2^{-\Delta \Delta \mathrm{Ct}}\right)$ (Pfaffl, 2001) method and expressed as a fold change respective to the control. Each sample was analyzed in triplicate. The primer sequences used in this study are listed in Table I.

Statistical analysis. Data are presented as the mean \pm SD. Significant differences between non-load and stretch groups (shown in Figs. 2-4) were determined using Student's t-test. $\mathrm{P}<0.05$ denotes statistical significance.

\section{Results}

Identification of isolated BMSCs. To investigate the characterization of BMSCs, the expression of antigens on the cell 

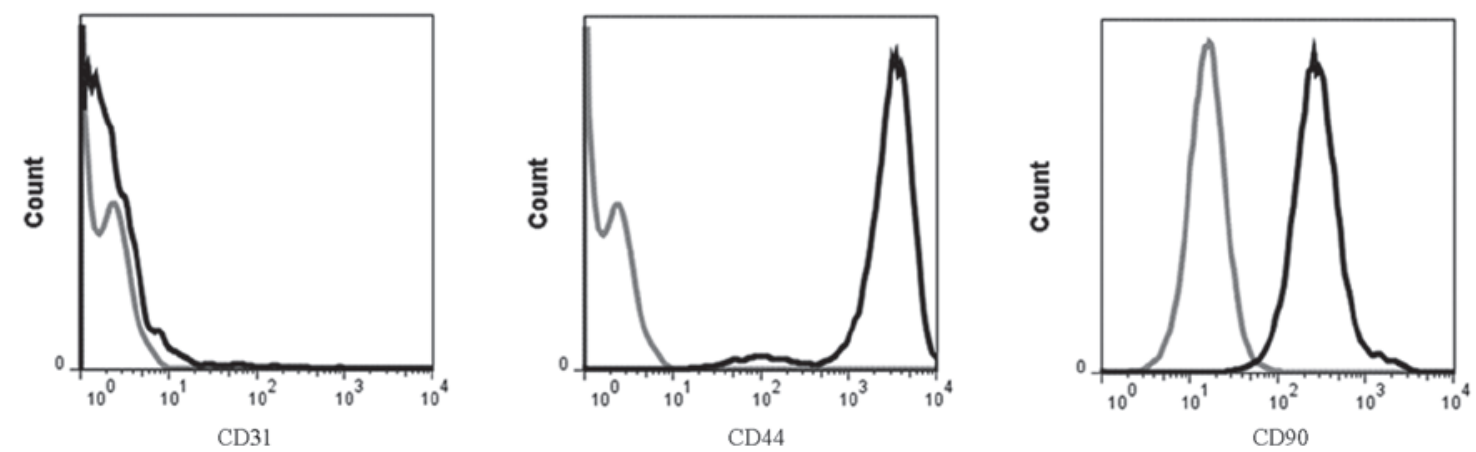

Figure 1. Flow cytometric analysis of antigen expression of bone mesenchymal stem cells. The positive rates of CD31, CD44 and CD90 were 1.5, 72.0 and $70.8 \%$, respectively.

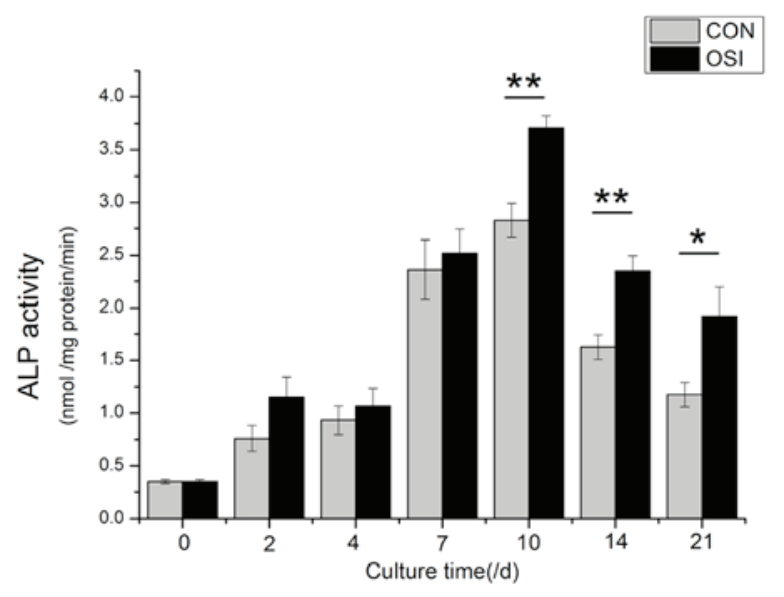

Figure 2. Time course of alkaline phosphatase (ALP) activity in bone mesenchymal stem cells (BMSCs). BMSCs in six-well plates $\left(1 \times 10^{5} \mathrm{cells} / \mathrm{cm}^{2}\right)$ were cultured with DMEM supplemented with 10\% FBS (control group) or DMEM with $10 \%$ FBS, $\beta$-glycerophosphate, dexamethasone and ascorbic acid. ALP activity of BMSCs was induced after 10 days of culture indicating osteoblastic differentiation. The values are the mean \pm standard deviation. There were significant differences of ${ }^{*} \mathrm{P}<0.05$ and ${ }^{* *} \mathrm{P}<0.01$. OSI, osteogenic induction group; $\mathrm{CON}$, non-osteogenic induction group.

surface was detected by flow cytometry. The positive rates of CD44 and CD90 were $72.0 \%$ and $70.8 \%$, respectively, but the positive rate of CD31 was only $1.5 \%$ (Fig. 1).

The quantitative analysis of ALP activity indicated that the cells cultured in the osteogenic medium demonstrated stronger ALP activity than those cultured in the growth medium, and reached a peak at Day 10 (Fig. 2).

Intermittent traction stretch promotes osteoblastic differentiation of BMSCs. In a previous study (10), we applied intermittent traction stretch stimulation to BMSCs and examined whether or not the progress of osteogenesis was switched on, as assessed by the presence of the osteoblast differentiation markers ALP, Collagen I, and OCN at the mRNA level. The results of those experiments showed that the stretch induces a time-dependent increase in the expression of osteoblastic genes and promoted the ALP activity (Fig. 3) at 7 days.

Expression of RANKL and OPG genes is affected by intermittent traction stretch. When BMSCs were exposed to intermittent traction stretch, the OPG mRNA level was
A

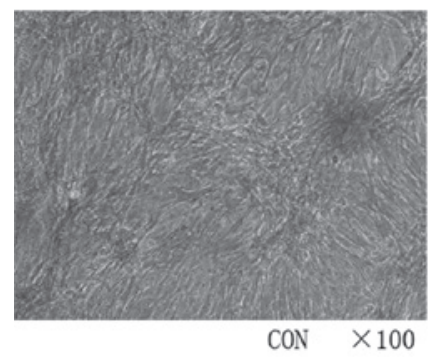

B

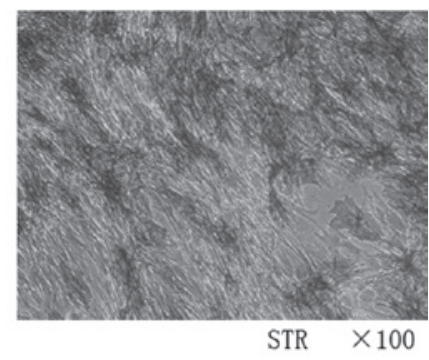

Figure 3. Intermittent traction stretch upregulated alkaline phosphatase activity of bone mesenchymal stem cells. (A) Non-loading group; (B) stretching group.

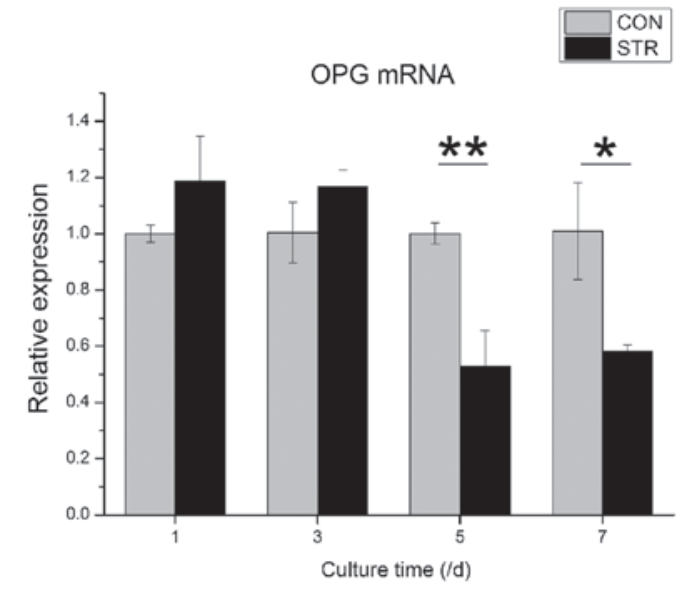

Figure 4. Expression of osteoprotegerin (OPG) mRNA affected by intermittent traction stretch. The values are the mean \pm standard deviation ( $\mathrm{P}<0.05$, ${ }^{* * *} \mathrm{P}<0.01$ as measured using an unpaired Student's t-test; $\left.\mathrm{n}=3\right) . \mathrm{CON}$, non-loading group; STR, stretching group.

increased slightly during the first 3 days. However, the OPG mRNA level decreased significantly at day $5(\mathrm{P}<0.01)$. At day 7, the OPG mRNA level of the stretching group was increased again, but remained lower than the non-loading group ( $\mathrm{P}<0.05$, Fig. 4$)$.

Dynamic stretch also showed different effects of RANKL mRNA expression at various time points. In contrast to OPG, RANKL gene expression was increased from the 1st day of loading to the 5 th day, followed by a decrease to approximately one fifth of the non-loading group $(\mathrm{P}<0.05$, Fig. 5). 


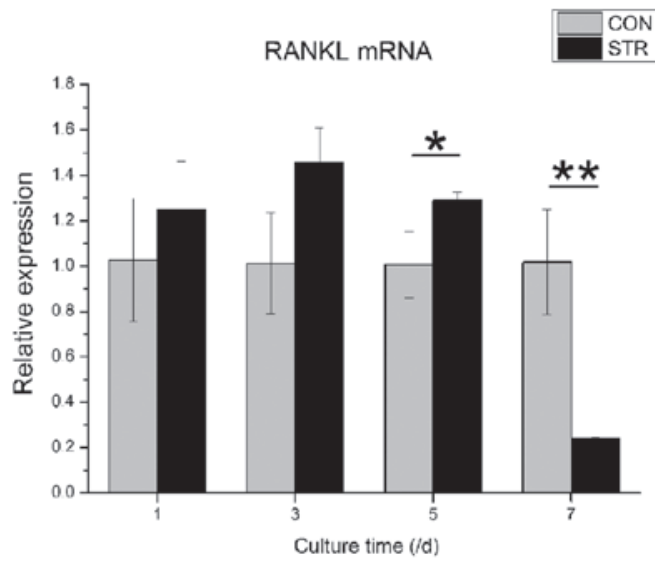

Figure 5. Expression of the receptor activator of nuclear factor- $\kappa \mathrm{B}$ ligand (RANKL) mRNA affected by intermittent traction stretch. The values are the mean \pm standard deviation $\left({ }^{*} \mathrm{P}<0.05,{ }^{* *} \mathrm{P}<0.01\right.$ as measured using an unpaired Student's t-test; $\mathrm{n}=3$ ). CON, non-loading group; STR, stretching group.

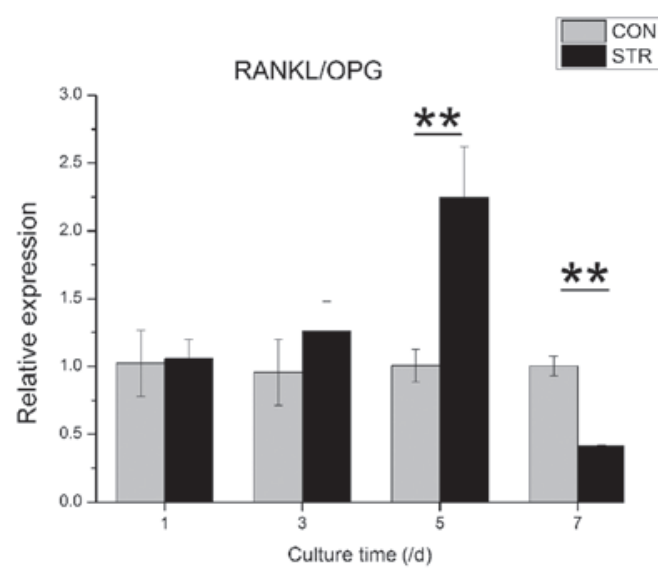

Figure 6. Receptor activator of nuclear factor- $\kappa \mathrm{B}$ ligand (RANKL)/ osteoprotegerin (OPG) ratio of bone mesenchymal stem cells affected by intermittent traction stretch. The values are the mean \pm standard deviation $\left({ }^{*} \mathrm{P}<0.05,{ }^{* *} \mathrm{P}<0.01\right.$ as measured using an unpaired Student's t-test; $\left.\mathrm{n}=3\right)$. CON, non-loading group; STR, stretching group.

RANKL/OPG ratio of BMSCs is affected by intermittent traction stretch. The RANKL/OPG ratio was increased gradually when BMSCs were exposed to dynamic intermittent traction stretch, and reached a climax (2.2-fold) at Day $5(\mathrm{P}<0.01)$. Of note is that the ratio was reduced to more than half at Day 7 ( $\mathrm{P}<0.01$, Fig. 6).

\section{Discussion}

Our previous studies have demonstrated that intermittent traction stretch could promote osteoblastic differentiation of BMSCs via the ERK-activated Cbfa1 signaling pathway (10). In the present study, we have shown that intermittent traction stretch affects the cytokine expression and production regulating osteoclastogenesis during the osteoblastogenesis process of BMSCs. Although several studies (10-12) have demonstrated that mechanical stress induces osteoblastic differentiation of osteoblastic cell lines, this is the first study to investigate the effects of intermittent traction stretch on osteoclastic-related cytokine production in BMSCs. Our results showed that intermittent traction stretch induced RANKL and OPG mRNA expression in a time-dependent manner and altered the RANKL/OPG ratio at different time points, initially upregulating the RANKL/OPG ratio and then downregulating it. This suggests that in the process of osteoblastogenesis of BMSCs induced by mechanical stretch, osteoclastic-related cytokine is simultaneously activated leading to the initiation of osteoclast activation. Nevertheless, osteoblastic differentiation of BMSCs was continuously enhanced by appropriate mechanical stretch, the osteoclastic-related cytokine was downregulated and then bone was deposited in the tension side.

Different behaviors of RANKL and OPG expression in various cells under different mechanical conditions have been reported. In the human osteoblastic cell line MG-63, OPG production increased subsequent to exposure of a cyclic strain; however, RANKL expression was not altered (12). A tensile strain upregulated OPG expression of mouse osteoblastic MC3T3-E1 cells and downregulated RANKL expression. Moreover, hydrostatic pressure changed the ratio of RANKL/OPG in favor of RANKL both at the mRNA and protein levels in a magnitude- and time-dependent manner (13). These studies all demonstrated that mechanical stress suppressed osteoclastogenesis and inhibited bone resorption. However, the exact relative behaviors of RANKL and OPG differ slightly, which is possibly due to the differences in mechanical conditions (strength, time and quality) and types of cells in vitro. Of note is that during tooth movement in vivo, mechanical stress stimulates osteocytes to express OPN in order to initiate bone remodeling (14-16), resulting in bone resorption and apposition on opposite sides of the tooth.

Similarly, it has been previously suggested that in response to mechanical forces, osteocytes regulate the recruitment of osteoclasts to sites of bone resorption by inducing the expression of RANKL by osteoblastic cells in the local micro-environment (17). This finding mainly consists of localization of RANKL-expression cells in bone sections $(18,19)$ and detection of RANKL expression in cells isolated from rodent calvarias, which are rich in osteoblast progenitors (7). However, the results revealed in those studies are inconsistent with each other. For instance, some studies detected RANKL in osteocytes while others did not detect RANKL. Moreover, calvarial preparations are heterogeneous and contain other cell types in addition to osteoblast precursors $(20,21)$, some of which may be sources of RANKL. Furthermore, the conditional ablation of osteocalcin-expressing cells in mice did not alter osteoclast number and function (22), indicating that mature osteoblasts and their immediate precursors may not be an essential source of RANKL in bone. Thus, while osteoblast precursors may be an important source of RANKL, this has not yet been proven experimentally. In addition, increased bone formation does not result in increased bone resorption in available models $(23,24)$. These latter studies dissociate the well-elucidated role of cells of the osteoblastic lineage in osteoclastogenesis from the potential influence of bone formation on bone resorption. Taken together, this evidence suggests that cells that support osteoclast formation may share a common progenitor with cells that differentiate into matrix-synthesizing osteoblasts. If this were found to be the case, it would provide strong support for the hypothesis that 
the balance in bone turnover is maintained, in part, by associating osteoclast and osteoblast differentiation.

The bone remodeling cycle could be altered by a number of stimulations, including mechanical stress. Our results indicate that mechanical stretch induced osteoclastogenesis to a certain extent. In the bone remodeling process, osteoclastogenesis and bone resorption occur initially and subsequently bone formation proceeds. In this study, we investigated the effect of mechanical stretch on RANKL and OPG production in BMSCs over a period of time. Our results seem to represent the initial and turning stage of the bone remodeling cycle. Moreover, in the experiment with a model loaded with long-term mechanical stress $(25,26)$, osteogenic marker upregulation was observed in osteoblasts loaded with mechanical stress compared with that for non-loaded osteoblasts.

A new mode of osteoclast-osteoblast communication has been identified in which Sema4D secreted by osteoclasts acts as a ligand and binds its receptor, Plexin-B1, on osteoblasts, thus suppressing their differentiation (27). The osteoclasts were shown to highly express Sema4D, and that expression is further increased during RANKL-induced osteoclastogenesis. When the number of osteoblastic cells increases, more RANKL is available to stimulate osteoclastogenesis, which in turn produces more Sema4D to reduce osteoblast differentiation, thereby serving as a negative feedback loop to balance the supply of osteoclasts and osteoblasts. As Sema4D plays an essential role in bone remodeling, it is necessary to investigate Sema4D for analysis of the molecular mechanism in bone remodeling induced by mechanical stress.

In conclusion, we have demonstrated that intermittent traction stretch regulated the production of RANKL in a time-dependent manner and initially changed the RANKL/OPG ratio in favor of RANKL in BMSCs downregulating the RANKL/OPG ratio later. These results suggest that BMSCs play an important role in cytokine production in response to mechanical stress and that orthodontic force may support the maintenance of mandible bone homeostasis by activating bone remodeling through osteoclastogenesis in vivo.

\section{Acknowledgements}

This study was supported by the National Nature Science Foundation of China (grant nos. 30901698 and 10972142), Collaborative Foundation of Medical and Engineering Science of Shanghai Jiaotong University (YG2012MS40), Key Basic Research Foundation of the Shanghai Committee of Science and Technology, China (12JC1405700), Program for Innovative Research Team of Shanghai Municipal Education Commission.

\section{References}

1. Heinonen A, Sievanen H, Kyrolainen H, et al: Mineral mass, size, and estimated mechanical strength of triple jumpers' lower limb. Bone 29: 279-285, 2001.

2. Dunphy L: Contemporary orthodontics, 5th edition. Br Dent J 213: 258, 2012

3. Krishnan V and Davidovitch Z: Cellular, molecular, and tissue-level reactions to orthodontic force. Am J Orthod Dentofacial Orthop 129: e1-e32, 2006.
4. Masella RS and Meister M: Current concepts in the biology of orthodontic tooth movement. Am J Orthod Dentofacial Orthop 129: 458-468, 2006.

5. Davidovitch Z: Tooth movement. Crit Rev Oral Biol Med 2: 411-450, 1991.

6. Koka S and Reinhardt RA: Periodontal pathogen-related stimulation indicates unique phenotype of primary cultured human fibroblasts from gingiva and periodontal ligament: implications for oral health disease. J Prosthet Dent 77: 191-196, 1997.

7. Lacey DL, Timms E, Tan HL, et al: Osteoprotegerin ligand is a cytokine that regulates osteoclast differentiation and activation. Cell 93: 165-176, 1998.

8. Teitelbaum SL: Osteoclasts: what do they do and how do they do it? Am J Pathol 170: 427-435, 2007.

9. Boyle WJ, Simonet WS and Lacey DL: Osteoclast differentiation and activation. Nature 423: 337-342, 2003.

10. Wu Y, Zhang X, Zhang $\mathrm{P}$, et al: Intermittent traction stretch promotes the osteoblastic differentiation of bone mesenchymal stem cells by the ERK1/2-activated Cbfa1 pathway. Connect Tissue Res 53: 451-459, 2012.

11. Zhang $\mathrm{P}, \mathrm{Wu} \mathrm{Y}$, Jiang Z, et al: Osteogenic response of mesenchymal stem cells to continuous mechanical strain is dependent on ERK1/2-Runx2 signaling. Int J Mol Med 29: 1083-1089, 2012.

12. Saunders MM, Taylor AF, Du C, et al: Mechanical stimulation effects on functional end effectors in osteoblastic MG-63 cells. J Biomech 39: 1419-1427, 2006.

13. Yamamoto $\mathrm{K}$, Yamamoto $\mathrm{T}$, Ichioka $\mathrm{H}$, et al: Effects of mechanical stress on cytokine production in mandible-derived osteoblasts. Oral Dis 17: 712-719, 2011.

14. Fujihara S, Yokozeki M, Oba Y, et al: Function and regulation of osteopontin in response to mechanical stress. J Bone Miner Res 21: 956-964, 2006.

15. Kuroda S, Balam TA, Sakai Y, et al: Expression of osteopontin mRNA in odontoclasts revealed by in situ hybridization during experimental tooth movement in mice. J Bone Miner Metab 23: 110-113, 2005.

16. Terai K, Takano-Yamamoto T, Ohba Y, et al: Role of osteopontin in bone remodeling caused by mechanical stress. J Bone Miner Res 14: 839-849, 1999.

17. Kartsogiannis V, Zhou H, Horwood NJ, et al: Localization of RANKL (receptor activator of NF kappa B ligand) mRNA and protein in skeletal and extraskeletal tissues. Bone 25: 525-534, 1999.

18. Ikeda $\mathrm{T}$, Utsuyama $\mathrm{M}$ and Hirokawa $\mathrm{K}$ : Expression profiles of receptor activator of nuclear factor kappaB ligand, receptor activator of nuclear factor $\mathrm{kappaB}$, and osteoprotegerin messenger RNA in aged and ovariectomized rat bones. J Bone Miner Res 16: 1416-1425, 2001.

19. Silvestrini G, Ballanti P, Patacchioli F, et al: Detection of osteoprotegerin (OPG) and its ligand (RANKL) mRNA and protein in femur and tibia of the rat. J Mol Histol 36: 59-67, 2005.

20. Guenther HL, Hofstetter W, Stutzer A, et al: Evidence for heterogeneity of the osteoblastic phenotype determined with clonal rat bone cells established from transforming growth factor-beta-induced cell colonies grown anchorage independently in semisolid medium. Endocrinology 125: 2092-2102, 1989.

21. Kalajzic I, Staal A, Yang WP, et al: Expression profile of osteoblast lineage at defined stages of differentiation. J Biol Chem 280: 24618-24626, 2005.

22. Corral DA, Amling M, Priemel M, et al: Dissociation between bone resorption and bone formation in osteopenic transgenic mice. Proc Natl Acad Sci USA 95: 13835-13840, 1998.

23. Ducy $\mathrm{P}$, Amling M, Takeda S, et al: Leptin inhibits bone formation through a hypothalamic relay: a central control of bone mass. Cell 100: 197-207, 2000.

24. Jochum W, David JP, Elliott C, et al: Increased bone formation and osteosclerosis in mice overexpressing the transcription factor Fra-1. Nat Med 6: 980-984, 2000.

25. Liu J, Zhao Z, Zou L, et al: Pressure-loaded MSCs during early osteodifferentiation promote osteoclastogenesis by increase of RANKL/OPG ratio. Ann Biomed Eng 37: 794-802, 2009.

26. Zhu J, Zhang X, Wang C, et al: Different magnitudes of tensile strain induce human osteoblasts differentiation associated with the activation of ERK1/2 phosphorylation. Int J Mol Sci 9: 2322-2332, 2008

27. Negishi-Koga T, Shinohara M, Komatsu N, et al: Suppression of bone formation by osteoclastic expression of semaphorin 4D. Nat Med 17: 1473-1480, 2011. 